\title{
El Acuerdo Transatlántico de Comercio e Inversiones (Transatlantic Trade and Investment Partnership, TTIP) entre la Unión Europea y Estados Unidos: informe provisional de su contexto y repercusiones*,**
}

\author{
The Transatlantic Trade and Investment Partnership (TTIP) \\ between the European Union and the United States: \\ Provisional Report on its Context and Impact
}

Joaquín Roy

University of Miami

Sumario: I. Trasfondo. 1. Paradojas. 2. Temas, desacuerdos, sugerencias. 3. Recomendaciones. 4. Síntomas. 5. Detalles comunes. 6. Preguntas cruciales.-II. La visión desde Europa. 1. El prisma europeo. 2. Reticencias de las posiciones españolas. - III. Percepción de Estados Unidos. 1. El factor intrahistórico. 2. Entre la visión gubernamental y la analítica.IV. Repercusiones en América Latina.-V. Brevísima y provisional conclusión.

Resumen: El anuncio de las negociaciones para un Acuerdo Transatlántico de Comercio e Inversiones (Transatlantic Trade and Investment Partnership, TTIP) en-

* Recibido el 7 de enero de 2015, aceptado el 19 de enero de 2015

** Este trabajo es una consecuencia de la preparación, ejecución y repercusiones de un seminario celebrado en la Universidad de Miami, bajo la cobertura doble del Centro de Excelencia de la Unión Europea y la Cátedra Jean Monnet, según se reflejó en una compilación: ROY, J. and DOMÍNGUEZ, R. (eds.). The Transatlantic Trade and Investment Partnership between the European Union and the United States. Miami-Florida European Union Center/ Jean Monnet Chair, 2014. http://www.as.miami.edu/media/college-of-arts-and-sciences/ content-assets/euc/docs/books/TTIP.pdf. Además de la documentación aportada, el presente estudio refleja en síntesis global los comentarios recibidos antes y después de una decena de seminarios, conferencias y presentaciones del libro, celebrados en ambos continentes a lo largo de 2014. Entre las ciudades sedes de estas actividades, se destacan las siguientes: Recife (Universidad Federal, Pernambuco, Brasil), Miami, México DF (El Colegio de México, UNAM, ITAM), Chicago Ill (Latin American Studies Association, LASA), Bucharest (National University), Villahermosa, Tabasco (Mexico, AMEI), San Miguel de Allende, Lleida, Barcelona (Universitat Ramón Llull, Facultats de Dret y Economía), Universitat Pompeu Fabra, Bilbao (Universidad de Deusto), Valencia (Facultat de Dret), y Zaragoza (Facultat de Derecho). Para la compilación y organización de datos bibliográficos, se agradece la colaboración especial de Nilda García, Jorge Smith y Diana Soller. 
tre la Unión Europea y Estados Unidos ha presentado un reto analítico notable. A pesar de que el proyecto tiene una larga tradición y los dos socios son aliados naturales en diversos frentes de las relaciones internacionales, numerosos sectores presentan dificultades para su buena resolución. Aunque la reducción de los aranceles hasta el máximo no presenta mayor dificultad, los temas de las regulaciones, mutuo reconocimiento y especificaciones nacionales se imponen como obstáculos para una negociación que no va a ser fácil ni veloz, y que se puede extender más allá de 2015. Este análisis delinea las diversas percepciones a ambos lados del Atlántico y añade algunas especulaciones sobre el impacto en América Latina.

Palabras clave: Estados Unidos. Unión Europea. Comercio. Inversiones. Aranceles. Reglamentaciones. España. América Latina.

Abstract: The announcement of the negotiations for a Transatlantic Trade and Investment Partnership, TTIP) between the European Union and the United States has presented a notable analytical challenge. In spite of the fact that the project has a long tradition and the two partners are natural allies in different fronts of international relations, numerous sectors present difficulties for a good resolution. While tariff maximum reduction will not be an obstacle, regulations, mutual recognition and national specifications and protections will pose challenges for a negotiation that is not going to be easy, nor fast -it could be extended well after 2015. This study outlines the diverse perceptions on both sides of the Atlantic and adds some speculative comments about the impact on Latin America.

Keywords: United States. European Union. Trade. Investments. Tariffs. Regulations. Spain. Latin America.

\section{Trasfondo}

\section{Paradojas}

Tratar el tema del Acuerdo del Partenariado Trans-Atlántico de Comercio e Inversiones (Trans-Atlantic Trade and Investment Partnership, TTIP) entre la Unión Europea y Estados Unidos es una tarea lastrada por varias dificultades. En primer lugar, implica una agenda de inspección sobre un proyecto que apenas ha comenzado a desarrollarse, y del que se tienen dudas de su consecución. Aunque el cuerpo de análisis apresurados es respetable, la investigación rigurosa no está todavía respaldada por una sólida base documental, más allá de los lineamientos generales acordados por ambas partes contratantes acerca del terreno básico de las negociaciones y un calendario sucinto de reuniones negociadoras en ambos continentes que han estado cumpliendo su agenda. Al mismo tiempo, con la excepción de puntuales declaraciones e informes escuetos de instituciones, líderes y funcionarios 
europeos $^{1}$ y norteamericanos, ${ }^{2}$ al finalizar 2014 no existía un legado de doctrina que reflejara posiciones fijas en ambos socios. Simultáneamente, el ambiente del proceso se ha visto pespunteado por observaciones urgentes y opiniones de expertos que permiten tener una idea más coherente acerca del

1 Para muestras de fuentes europeas: BBC News Europe, «EU reveals US trade talks agenda in key TTIP document», 9 de octubre de 2014, http://www.bbc.com/news/worldeurope-29551520301952; European Commission, «The Transatlantic Trade and Investment Partnership», 5 de mayo de 2014, http://ec.europa.eu/trade/policy/in-focus/ttip/; EUROPEAN COMMISSION, TTIP explained, http://trade.ec.europa.eu/doclib/docs/2014/may/tradoc_152462.pdf; COUNCIL OF THE EUROPEAN UNION, Declassification, ST 11103/13 Resterint UE/EU Restricted, 17 June 2013-9 October 2014, Directives for the negotiations on the Transatlantic Trade and Investment Partnership between the European Union and the United States, http://data.consilium.europa.eu/doc/document/ST-11103-2013-DCL-1/en/pdf

Como ejemplos de declaraciones positivas, véanse: DE GUCH, K., «El estímulo más barato que usted pueda imaginar», Política Exterior, mayo-junio 2013, pp. 50-56; DE GUCHT, K., «The Transatlantic Trade and Investment Partnership: Global Impacts,» European Commission Delegation of the European Union to the United States, Brussels, Op-Ed by Ambassador Vale De Almeida in the Hill: «More Trade Brings More Jobs», 12 de diciembre de 2013, http://www .euintheus.org/press-media/op-ed-by-ambassador-vale-de-almeida-in-thehill-more-trade-brings-more-jobs/; Delegation of the European Union to the United States, «Transatlantic Trade and Investment Partnership (TTIP). Creating Jobs, Boosting Exports, and Investing in the Economy of Tomorrow», TTIP Fact Sheet, http://www.consulfrancemiami.org/IMG/pdf/TTIP_Publicatiopn_85x11in_High_res.pdf;

Ahora bien, en el seno de las instituciones europeas y de algunos de sus partidos de alcance continental se fueron detectando focos de advertencia acerca de las dificultades del proceso de negociación: EUOBSERVER, "Scaremongering” threatens trade deal, US ambassador warns MEPs», 04 de septiembre de 2014, http://euobserver.com/news/125459; EUOBSERVER, «The rollback of transparency», 23 de septiembre de 2014, http:// euobserver.com/opinion/125718; EURACTIV, «Green party leaks confidential TTIP paper», 28 de marzo de 2014, http://www .euractiv.com/sections/trade-industry/green-partyleaks-confidential-ttip-paper-300760; EURACTIV, «Schulz on TTIP: There will be no secret negotiations», 8 de mayo de 2014. http://www.euractiv.com/sections/trade-industry/ schulz-ttip-there-will-be-no-secret-negotiations-301952; EURACTIV, «Green party leaks confidential TTIP paper», 28 de marzo de 2014, http://www.euractiv.com/sections/tradeindustry/green-party-leaks-confidential-ttip-paper-300760; European Parliament. «The Transatlantic Trade and Investment Partnership (TTIP) and Labour». http://www.europarl.europa. eu/meetdocs/2014_2019/documents/empl/dv/empl20141201_ttip_briefing_/empl20141201_ ttip_briefing_en.pdf

${ }^{2}$ Para una selección de las declaraciones del gobierno norteamericano, véanse: OFFICE OF THE UNITED STATES TRADE REPRESENTATIVE, «U.S. International Trade Commission Releases Report on How T-TIP Will Benefit Small Businesses», Press Release, 28 de marzo de 2014, http://www.ustr.gov/about-us/press-office/press-releases/2014/ March/USITC-Releases-Report-How-TTIP-Will-Benefit-Small-Business; OFFICE OF THE UNITED STATES TRADE REPRESENTATIVE, «White House Fact Sheet: Transatlantic Trade and Investment Partnership (TTIP)», junio de 2013, http://www.ustr.gov/about-us/ press-office/fact-sheets/2013/june/wh-ttip; OFFICE OF THE UNITED STATES TRADE REPRESENTATIVE, «Transatlantic Trade and Investment Partnership». 10 de mayo de 2014. http://www.ustr.gov/ttip 
camino a recorrer y ciertas predicciones sobre los obstáculos hallados o por encontrar que influirán en el resultado final.

Por otra parte, el proceso se ha insertado en una época de la vida reciente de ambos socios que está larvada de incógnitas sobre la actuación, liderazgo y autodefinición de su propio papel en el mundo. A pesar de las declaraciones de la dirigencia de ambas partes, importantes incógnitas dominan el escenario. Destacan, entre ellas, una fundamental: ¿por qué se decidió en 2013 retomar un proyecto que, curiosamente, siempre ha tenido una lógica que ha sido recordada durante varias décadas? No se olvide que el proyecto se entabla entre dos entes de perfil contrastivo (un estado federal y una asociación de estados con una ambición de serlo algún día) que han sido y son dominadores del panorama mundial y que comparten una serie de valores con una intensidad formal superior a la que tienen o pueden tener otras posibles coaliciones.

En concreto, se especula acerca de las verdaderas motivaciones del proyecto. ¿Para qué? Más que los datos y detalles económicos, resultan intrigantes numerosas dimensiones de su contexto social y político, por no hablar del más ambicioso geopolítico. Curiosamente, la planeada «alianza» se lanza frecuentemente en unos momentos en que ambas partes pasan por una etapa, individual y colectiva, que universalmente es calificada de crisis, no solamente económica sino también de identidad, no solamente interior sino de actividad (delicada y afectada de temas cruciales de seguridad) hacia el exterior.

Estados Unidos parece estar experimentando una fase vital que confirmaría su aparente contradicción que revela una variante de su históricamente tradicional aislacionismo. No ha renunciado a su papel primigenio pero ha dado muestras de retraimiento y prudencia. Este es un diagnóstico que sorprende a los comentaristas que se fían de la imagen ofrecida por las numerosas etapas intervencionistas que consolidaron su reputación imperialista. Pero la innata inclinación de la sociedad norteamericana es originariamente aislacionista, reflejada en la influencia inicial del pensamiento de Thomas Jefferson. Esta seña de identidad contrasta paradójicamente con un práctico historial que notablemente se dirige en la dirección contraria, la intervencionista.

En sus entrañas, el bando republicano, aunque pudiera reflejar los intereses del empresariado más abierto a las innovaciones exteriores, puede destacar en generar los principales obstáculos para la aprobación del TTIP. Esto puede suceder por la confluencia de dos factores. Uno sería la exigencia y exageradas expectativas de máxima apertura y mínimos obstáculos para numerosos productos, una percepción que chocaría con las reticencias y la prudencia de los sectores europeos. El otro estaría representado por el populismo protagonizado por el Tea Party y sectores afines, insertados en el 
seno republicano, siempre en búsqueda de temática con la que se pueda erosionar el liderazgo tradicional.

En el campo demócrata, las reacciones pueden presentar un contraste curioso. Por un lado, numerosos intereses de los sindicatos se sienten intrigados acerca de las posibles ventajas que se pueden derivar de la adopción de estándares y reglamentaciones europeos que puedan proteger los intereses laborales de la clase trabajadora, golpeada por la crisis. Pero también se debe contar con la fuerza de resistencia de ese mismo sector, tradicionalmente celoso del poder derivado del proteccionismo

En el entramado institucional norteamericano habrá que prestar atención a lo que se etiqueta como la «dispersión del poder», innato en el sistema federal, no solamente en lo que atañe a la competencia de los estados entre sí, sino también a la rivalidad entre el ejecutivo y el legislativo. Obama ha seguido estando dañado por el lastre de no contar con el anhelado privilegio de fast track.

Otro obstáculo, compartido por diversos sectores partidistas, es la obsesión genuinamente norteamericana de superar a Europa, o por lo menos en expresar una mezcla de ignorancia y desdén. El reflejo más claro de este sentimiento es la doctrina del excepcionalismo norteamericano, la autoconsideración de sentirse como un «faro de luz en lo alto de la colina». Obsérvese que esta certeza innata se refleja en expresiones tan populares como que «no hay nada por encima del Tribunal Supremo» y el rechazo de la supranacionalidad, lo que contrasta contundentemente con la normalidad (luego de notable esfuerzo) europea de la integración continental.

\section{Temas, desacuerdos, sugerencias}

Como socios privilegiados que se respetan y confían mutuamente, Washington y la UE todavía tienen desacuerdos y desconfianzas no solamente presentes en detalles económicos y medioambientales (subsidios agrícolas, procesamiento de alimentos, regímenes de comercialización del plátano), sino también sobre temas sensiblemente fundamentales. En este terreno, diversas dimensiones generales y concretas están entrelazadas. Algunas fuentes de conflictos se originan en terrenos tan diversos como los temas de seguridad nacional, la protección de los derechos humanos en todo el mundo, el embargo de armas contra China, los subsidios para Boeing y Airbus, los presupuestos de defensa, y los concursos de los contratos para la producción de sistemas armamentísticos. Como consecuencia de la llamada «guerra contra el terror», algunos estados europeos se vieron involucrados en la transferencia ilegítima de detenidos y su ulterior prisión, acusados de 
ser cómplices de acciones criminales. Esta situación ha creado controversias internas en algunos estados afectados, cuyos gobiernos se han visto presionados por la oposición interna, acusados de plegarse a las expectativas y demandas de Washington. Los dos mandatos de George W. Bush se vieron plagados de desacuerdos.

En el caso de los principios que son considerados indiscutibles e innegociables en Europa, pero que en Estados Unidos están condicionados por las legislaciones de los estados individuales y las decisiones del Tribunal Supremo, existe un desacuerdo frontal con respecto a la pena máxima. Entre las consecuencias colaterales, destaca la prohibición de deportación de presuntos criminales a Estados Unidos si existe la posibilidad de su ulterior ejecución. Otro tema de desacuerdo es el rechazo de Estados Unidos a reconocer la jurisdicción de la Corte Internacional de Justicia Criminal, debido a que Washington teme que los soldados norteamericanos puedan ser objeto de juicios por motivaciones políticas.

Como consecuencia de las restricciones de ingreso en territorio norteamericano después de los ataques de 2001, Estados Unidos indicó algunas veces su intención de exigir visados a todos los ciudadanos de la UE (o de algunos estados especiales). A pesar de que Bruselas se expresó con amenazas de represalias, ambas decisiones nunca se materializaron. Si bien Estados Unidos y los estados europeos comparten la meta común de que se alcance un acuerdo para los territorios palestinos, ambas partes favorecen diferentes alternativas. En la práctica, Europa es más benévola que Washington ante las acciones palestinas, mientras que los norteamericanos justifican las acciones israelíes hasta el extremo de ejercer sistemáticamente un veto en el Consejo de Seguridad. Mientras Washington ha justificado la opción de un ataque «preventivo» contra Irán bajo la sospecha del uso de armas nucleares, los europeos prefieren la negociación diplomática. La guerra de Irak marcó la cima del desacuerdo entre los gobiernos europeos y Washington (con la excepción de Reino Unido y algunos estados especiales). La penúltima fuente de confrontación fue el descubrimiento de sistemas de espionaje ejecutados por Estados Unidos, sin discriminar entre aliados y adversarios.

\section{Recomendaciones}

En el amplio tema de las relaciones transatlánticas, cubierto por una amplia literatura durante un largo medio siglo, destaca la agenda de las recomendaciones ofrecidas por especialistas académicos, comisiones, centros de reflexión, lobbies, reuniones ad-hoc, seminarios y conferencias de diverso formato y extensión. 
Un ejemplo todavía válido es un informe colectivo forjado por el Center of Transatlantic Relations de la Johns Hopkins University (SAIS), ${ }^{3}$ con la colaboración de una docena de los más prestigiosos think-tanks de ambos continentes. Especialistas europeos y norteamericanos examinaron el peligro de que el llamado «partenariado» transatlántico se difuminara sin apenas dejar rastro. Sin embargo, el legado del esfuerzo forjado a través de décadas todavía sobrevive en un mundo en el que las fronteras nacionales e internacionales están borrosas.

La realidad es que cuando hay un desacuerdo, ambos actores no pueden lograr en aislamiento las metas que se propusieron en común. En rigor, la evidencia muestra que no existe una coalición de alcance planetario que funcione satisfactoriamente. Europa y Estados Unidos no pueden resolver los problemas que trascienden sus propias fronteras. En asociación con otros agentes, pueden lograr el éxito. Juntos, norteamericanos y europeos necesitan posicionar sus economías, conservar una Europa libre y abierta, encarar los conflictos eficazmente, oponerse a la proliferación de las armas nucleares, y preservar el frágil medio ambiente planetario.

Sin embargo, hay todavía una carencia de acuerdo acerca de la naturaleza de los retos, la capacidad de las instituciones, y las herramientas disponibles. Existe una necesidad de una sólida cooperación entre Estados Unidos y Europa con el fin de convertir la simple relación en un vínculo estratégico.

Para conseguir este éxito, se exponen una serie de recomendaciones. Por ejemplo, debiera forjarse un compromiso sólido de solidaridad transatlántica en las áreas de justicia, libertad y seguridad (el viejo «pilar» de la UE). Hay la urgente necesidad de reforzar la gobernanza económica, una vez parece que ha desaparecido la «vieja» y la «nueva» se halla en estado precario debido al debilitamiento de la economía europea y el surgimiento de la influencia de los poderes emergentes, principal el núcleo compuesto por los llamados BRICS (Brasil, Rusia, India, China y Sudáfrica).

Existe también la necesidad de una coalición en el tema de los recursos de energías renovables y las alternativas disponibles. En suma, hay la necesidad de la creación de un verdadero mercado transatlántico, libre de obstáculos legales y tarifarios, un compromiso de los intereses comerciales de Estados Unidos para poder operar en Europa. Finalmente, es necesaria la existencia de una «Europa completa, libre y en paz». Juntos, Europa y Estados Unidos podrán encarar, de la forma más eficaz, los conflictos bélicos que periódicamente estallan, y reforzar los esfuerzos para frenar la produc-

${ }^{3}$ HAMILTON, D. (ed.), Shoulder to Shoulder: Forging a Strategic US-EU Partnership, Center for Transatlantic Relations, Washington DC, 2010. 
ción de armas de destrucción masiva. Estos dos grandes poderes atlánticos con verdadera capacidad de asistencia al desarrollo debieran trabajar juntos para coordinar mejor su eficacia en el campo de la ayuda humanitaria.

Sin embargo, entre las conclusiones de estos argumentos, destacan algunas advertencias. Lamentablemente, su disposición para forjar una coalición débil generará obstáculos para llegar a la consecución de una sólida relación. Como consecuencia, este defecto provocará desafíos de otros poderes emergentes. Para evitar el error de experimentos precedentes, en lugar de inventar la rueda con la puesta en marcha de foros y mecanismos adicionales, debieran reforzarse los ya existentes (la OTAN, por ejemplo). En una forma más concreta, las relaciones inter-parlamentarias debieran ser institucionalizadas. Ambas partes debieran evitar implicaciones extraterritoriales con sus correspondientes legislaciones que producirían innecesarios conflictos.

A pesar de los buenos deseos ya existentes, debiera prestarse atención a los obstáculos presentados por el populismo y el radicalismo ideológico que han aparecido en Europa y Estados Unidos. Estos nuevos «actores» representan una competencia desleal. La información incompleta generalmente impele a los ciudadanos a elegir líderes que se han presentado como «nacionalistas».

En un nivel más profundo, este informe reconoce la ruptura de la relación entre Estados Unidos y la Unión Europea procedentes de dos orígenes, ambos peligrosos y preocupantes. El primero procede del aumento de la dependencia de ambos socios de la energía del exterior (pero Estados Unidos parece ya estar disfrutando de liberalizarse de esta necesidad), un defecto que produciría conflictos y competencia para el control de acceso de zonas de producción. El segundo, íntimamente relacionado con el primero, deriva del desequilibrio militar entre ambas partes, y la base ideológica de tal contraste. Mientras Europa ha insistido en el progresivo desarme, Estados Unidos todavía posee la más formidable fuerza armada del planeta. Incluso al revisar la capacidad militar de Francia y Reino Unido, los dos estados europeos que todavía presumen de una defensa autónoma, ninguno puede compararse con cualquiera de las ramas militares de Estados Unidos.

A la vista de todos estos aspectos, numerosos analistas han propuesto la tesis de que en la década precedente, la ausencia de acuerdo no se debe a motivaciones simplemente transitorias. Por el contrario, responden a síntomas más profundos que reflejan innatas diferencias de personalidad. En suma, los europeos vendrían de Venus, mientras los norteamericanos serían descendientes de $\mathrm{Marte}^{4}$. Mientras Europa se sentiría más inclinada a cons-

${ }^{4}$ Véase este libro clásico: KAGAN, R., Of Paradise and Power, Alfred Knoft, New York, 2003. 
truir una paz perpetua por medio de la negociación y la diplomacia, Estados Unidos vería en un mundo hobbesiano un mensaje de fuerza como garantía de estabilidad y supervivencia. Lo que todavía es objeto de debate es cuál de estas visiones tiene más posibilidades de solucionar los serios problemas que el mundo encara. En consecuencia, mucho depende de la necesaria cooperación de estas dos visiones. En gran manera, el proyecto del TTIP es el resultado lógico de reforzar ese consenso general, sobre todo en época de crisis.

\section{Sintomas}

Según predicciones predominantes, la evolución del proceso del TTIP puede tener por lo menos tres resoluciones. Estas solamente comparten un par de características comunes e incuestionables: el trámite no va a ser fácil, ni va a ser veloz. Por el contrario, será largo y laborioso. En resumen, uno de los escenarios estaría ocupado por un acuerdo ambicioso, modélico de aplicación política a un complejo contenido económico, que revolucionaría el tejido comercial y de inversiones en el planeta y dejaría a las dos potencias en situación ventajosa para encarar el futuro. El segundo escenario sería más modesto, aunque tendría una resolución positiva que diera a cada uno de los socios una razón para sentirse satisfechos. Pero habría sido el resultado de un acuerdo de mínimos, con multitud de excepciones y zonas prohibidas, que darían la sensación de haber forjado una especie de queso de Gruyere, de la variedad que presume de múltiples agujeros. El tercer escenario sería de fracaso por no haber podido lograr un básico acuerdo y quedar tan enterrado como el Area de Libre Comercio de las Américas (FTAA, o ALCA), de infausta memoria, inaugurado justamente hace veinte años en Miami. ${ }^{5}$

Sobre la suposición de que el resultado va a quedar entre la realidad primera y la segunda, es ya comprensible entender que esta variante de negociaciones internacionales, sobre todo teniendo en cuenta el perfil de los contrayentes, máximas potencias mundiales, no va a dejar contentos a todos, aunque se consiga el éxito al mayor nivel. Lo que se acuerde, no traducirá todas las expectativas. Pero, pero al mismo tiempo el proyecto en sí es ya imparable. Esto se debe a que el capital político ya invertido es conside-

5 Véase nuestro análisis: ROY, J., «El Area de Libre Comercio de las Américas (ALCA): Su peculiaridad desde una perspectiva de la UE», ROY, J., et al., La Unión Europea y la Integración Regional: Perspectivas Comparadas y Lecciones para las Américas, Universidad Nacional de Tres de Febrero/ Consejo Argentino de Relaciones Internacionales, Buenos Aires, 2005, pp. 299-318. 
rable y esté forjado por dos socios naturales, tanto en historia y perfil social como en similitudes económicas y políticas.

El necesario análisis debiera subdividirse en por lo menos tres o cuatro partes diferenciadas, según los diferentes ángulos de perspectivas de los principales actores. Por lo tanto, aparte de unas preliminares pinceladas complementarias y comparativas, debiera prestarse atención por separado al contexto europeo y al norteamericano. Luego, en el terreno de la UE convendría meditar sobre algunas especificidades del caso español. Al otro lado del Atlántico, más allá de las repercusiones en el resto del globo, se recomendaría analizar, aunque sea de forma somera, el impacto que tanto las negociaciones tienen como los resultados futuros para los sistemas de integración latinoamericanos e inter-americanos, y sobre las propia negociaciones que ya están entabladas con los mismos contrayentes del acuerdo, Estados Unidos y la Unión Europea.

\section{Detalles comunes}

Resulta significativo, por otra parte, que los análisis que el TTIP ya ha generado, las negociaciones en marcha y las declaraciones que se suceden revelan no solamente detalles, sino que invitan a la meditación sobre dimensiones esenciales de ambos cómplices que frecuentemente se soslayan debido a las prisas provocadas por los acontecimientos. El primer detalle contrastivo entre los dos contrayentes en esta especie de matrimonio de conveniencia es que paradójicamente uno (Europa) parece adolecer de carencia de unidad política que sigue siendo su más serio lastre en capturar un necesario protagonismo en la escena mundial. El otro (Estados Unidos) puede por el contrario presumir de actuar unitariamente en el globo y estar accesible mediante un único teléfono (por usar la terminología atribuida a Henry Kissinger).

Pero ésa es solamente la apariencia, ya que en el terreno de las concretas negociaciones, la Unión Europea está representada por un ente supranacional por excelencia (la Comisión) que posee el monopolio de actuar en todo lo que implique al comercio exterior. Estados Unidos, por el contrario, adolece de una dispersión del poder en esa materia, ya que la principal autoridad es el Congreso, mientras el presidente nunca ha logrado manejar la ansiada fast track y apenas la ha solicitado por miedo de que el legislativo se la niegue. Esto no quiere decir que una vez la Comisión se ponga de acuerdo con su interlocutor norteamericano inicial (el Departamento de Comercio) todo estará atado y bien atado. Tanto el Consejo como el Parlamento europeos jugarán su papel decisivo.

En el terreno norteamericano, cualquiera que sea la senda tomada por el ejecutivo en la fase decisiva de los escarceos, hay que tener en cuenta que 
el sistema norteamericano partidario dista mucho de asemejarse al europeo. El Congreso no está compuesto por unos partidos tradicionales al modo europeo donde las consignas de las ejecutivas indican como se debe interpretar la partitura. En realidad, los partidos en Estados Unidos no existen como tales y solamente funcionan como coaliciones electorales. La disciplina de voto brilla por su ausencia. Senadores y congresistas solamente reconocen la lealtad hacia sus votantes más cercanos. Sus decisiones en temas tan complejos como el TTIP tienen más en cuenta las existencias diarias de los componentes de su circunscripción, las industrias que alojan, y la coyuntura económica y social del momento.

\section{Preguntas cruciales}

Como se alude más arriba, lo primero que uno se debe preguntar es: ¿por qué ahora?; ¿qué tiene de específico el escenario económico o geopolítico actual para haber impelido a estos dos socios a acordar el comienzo del proyecto? La primera respuesta lógica, si simplemente se observa la escena de los últimos años, es que la decisión está impelida por la generalizada crisis económica en el mundo, y muy especialmente en Estados Unidos y la UE.

Un poco más complicada es la siguiente pregunta: ¿para qué? Y más intrigante es una tercera: ¿contra quién? $\mathrm{O}$, por lo menos, ante quién se constituye esta especie de escudo que siempre son las alianzas. La respuesta preliminar, ya señalada por expertos, y nunca denegada explícitamente por los gobiernos y las propias instituciones de la UE, señala en la dirección de los BRICS. Su competencia en el mercado mundial y en el entramado geoestratégico ha captado la atención de Washington y Bruselas. ${ }^{6}$

El contexto y cierto perfil recuerda el escenario imperante en el momento en que la UE decidió aunar fuerzas con la Asociación Europea de Libre Comercio (EFTA, en inglés), su competidora sobre todo cuando el Reino Unido contumazmente se negaba a adherirse al más ambicioso proyecto europeo. Se formó entonces el Espacio Económico Europeo, rebautizado en otras regiones como la «fortaleza europea». Desde Estados Unidos se interpretó correctamente el reto y ése fue el origen de la for-

6 Véase: STEINBERG, F., «Underlying Geopolitical Motives of the TTIP: Leadership of the Transatlantic Axis», en ROY and DOMÍNGUEZ (editors). The TTIP: The Transatlantic Trade and Investment Partnership between the European Union and the United States, Miami-Florida European Union Center/Jean Monnet Chair, 2014, pp. 199-207, http://www. as.miami.edu/media/college-of-arts-and-sciences/content-assets/euc/docs/books/TTIP.pdf 
mación del Tratado de Libre Comercio de Norteamérica (TLC, o mejor conocido como NAFTA). Para usar la imaginería de la famosa serie fílmica de la «Guerra de las Galaxias» (Star Wars) que se estrenaba entonces, «el imperio contraatacaba». Se amplió así la sólida alianza que ya existía con Canadá, expandida a renglón seguido con la incorporación de México, que había agotado su conveniente estatus entre la dictadura del PRI y sus devaneos izquierdistas en el exterior tras la desaparición de la Guerra Fría. Similares cambios geopolíticos aparecen ahora. El siguiente paso fue la ampliación del concepto de NAFTA a cubrir toda la zona del hemisferio occidental con la puesta en marcha del ALCA en 1995, que terminó en descarrilamiento entre otras razones por las desavenencias con Brasil.

Tal como ya lo están reconociendo los diversos representantes tanto en sus propias negativas a admitir cambios en el statu quo de sus privilegios actuales como a señalar líneas rojas en los compromisos, habrá que meditar acerca del número y volumen de los temas que se consideran «sensibles» y que con el fin de llegar a unos acuerdos, quedarían fuera de los compromisos. Se generaría un acuerdo de mínimos, si bien pudiera considerarse como un logro político. Podría presentar resultados en un calendario razonable, pero lastrado por excepciones. Daría como resultado uno de los defectos mayores de un emblemático sistema de integración latinoamericano que se presentó desde su nacimiento con ambiciones de llegar a ser un «mercado común», tal como reza su nombre: el Mercado Común del Sur (MERCOSUR). Sus numerosas excepciones, conocidas también como «perforaciones», le han dado ya la imagen de un queso de Gruyere, que se debiera evitar en el caso del TTIP.

\section{La visión desde Europa}

\section{El prisma europeo}

En los medios oficiales de la UE se insiste en que el proceso, una vez cumplida la octava consulta y después de que se ofreció un resumen del estado de las negociaciones, en realidad la recta final comenzaría al finalizar el verano y ya entrado el otoño de 2014 y se esperaba que se extendiera no más allá de mediados de 2015. Desde Bruselas, la tarea más ardua ha sido saber en realidad qué persiguen los norteamericanos y cómo sus anhelos deben interpretarse en clave bicontinental. En ese brete, no se debe perder de vista que Estados Unidos está simultáneamente implicado en dos procesos, que provisionalmente pueden considerarse como complementarios: TTIP y 
el referente al Pacífico, el TIP.7 La clave consiste en internalizar que Washington trata de sacar lo mejor de cada proyecto. Desde la perspectiva europea, la atención se centrará en los aranceles sobre los productos cárnicos y las operaciones aplicables a la industria de automoción, sin perder de vista el tema de las reglamentaciones, sujetas al respeto al acervo comunitario, aspecto que Bruselas no abandonará. En cuanto a los sectores operativos, las Pymes y el terreno de la banca serán foco de preocupación, mientras las inversiones y los servicios se mantendrán como notables huesos duros de roer. La comunidad de análisis ha estado siguiendo las diversas dimensiones del proceso y ha estado emitiendo informes y advertencias de todo tipo, enfatizando la presencia de obstáculos, dudas y peligros. ${ }^{8}$ Algunos estados miembros han revelado su tradicional «excepcionalismo» nacional, como es el caso notorio de Francia, advirtiendo negativamente sobre el acuerdo. ${ }^{9}$

7 Véase un libro dedicado al tema: OROPEZA GARCÍA, A., El Acuerdo de Asociación Transpacífico: ¿bisagra o confrontación entre el Atlántico y el Pacífico?, UNAM, México DF, 2013.

${ }_{8}$ Para una muestra selectiva de los análisis europeos, y su reflejo en los servicios informativos especializados, véanse: BRIGINSHAW, J. et al., «Will the TTIP Happen?» Event Report, Chatham House, 9 de junio de 2014, http://www.chathamhouse.org/system/ files/field/field_document/20140609WillTTIPHappen.pdf; ERIXON, F. «Trans-Atlantic Trade and Investment Partnership and the European Parliament», ECIPE, 18 May 2014. http://www.voxeu .org/article/trans-atlantic-trade-and-investment-partnership-and-europeanparliament; EUOBSERVER, «EU-US trade deal could threaten GMO labels», 14 de noviembre de 2014, http://euobserver.com/tickers/126515; ABBOTT, R., «A Close Look at the Transatlantic Trade and Investment Partnership,» Friends of Europe, 25 de marzo de 2014, http://www.friendsofeurope.org/Contentnavigation/Publications/Libraryoverview/tabid/1186/ articleType/ArticleView/articleId/3717/A-close-look-at-the-Transatlantic-Trade-and-Investment-Partnership.aspx; ABBOTT, R., «A close look at the Transatlantic Trade and Investment Partnership», Europe's World, 28 de marzo de 2014, http://europesworld.org/2014/03/28/aclose-look-at-the-transatlantic-trade-and-investment-partnership/\#.U4unWChYpIt; EURACTIV, «Some little things you should know about TTIP», 19 de agosto de 2014, http://www. euractiv.com/sections/innovation-enterprise/some-little-things-you-should-know-about-ttip307818; EURACTIV, «CETA, TTIP: Global trade threatened by vested national interests», 27 de septiembre de 2014, http://www .euractiv.com/sections/trade-industry/ceta-ttip-globaltrade-threatened-vested-national-interests-308753; EURACTIV, «Commission swamped by 150,000 replies to TTIP consultation», 17 de julio de 2004, http://www.euractiv.com/sections/trade-industry/commission-swamped-150000-replies-ttip-consultation-303681; FOX, B., «Leaked paper: EU wants 'guaranteed' access to US oil and gas», EU Observer, 9 de julio de 2014; FOX, B., «What the leaked EU-Canada trade paper means for TTIP», EU Observer, 18 de agosto de 2014. http://euobserver.com/news/125270

9 Véanse algunas advertencias: BARBIERE, C., «French Government will not sign TTIP agreement in 2015», EURACTIV, 17 de noviembre de 2014, http://www.euractiv.com/sections/trade-society/french-government-will-not-sign-ttip-agreement-2015-310037; EURACTIV, «France's latest calls for TTIP transparency fall on deaf ears» 9 de septiembre de 2014 , http://www .euractiv.com/sections/trade-industry/frances-latest-calls-ttip-transparency-falldeaf-ears-308652; EURACTIV, «French Senators Strongly Attack EU-US Trade Deal», 13 
También han aparecido reticencias en el mismo corazón geográfico de las instituciones europeas y su estado sede principal, Bélgica. ${ }^{10}$

Como excepción a la modesta producción detallista, algunos estudios se han aventurado en el delineamiento de datos científicamente predecibles, aunque en numerosos casos sean en principio aplicables a las circunstancias de un solo país, como es el caso de Alemania, la economía más potente de Europa.$^{11}$ En suma, la reducción del proyecto que se limite a las barreras arancelarias no tendrá impacto significativo, mientras que si se amplía a la reducción de las reglamentaciones, el efecto ya sería más notable. Este esquema es similar a otros países de la UE. Mientras el comercio de la UE con los BRICS se reduciría modestamente, el de Estados Unidos con esos mismos países descendería en un 30\%. El intercambio comercial entre la Unión Europea y los países vecinos en el norte de África y en el propio este de la UE descendería en un 5\%. En los estados bálticos se observarían beneficios mediante la reducción de las barreras tarifarias con Estados Unidos. En general, algunos países de la UE recibirían mayores ventajas, como es el caso del Reino Unido y la cuenca mediterránea.

En cuanto al empleo, la reducción de barreras no estrictamente arancelarias aumentaría el número de puestos de trabajo en la UE. La pura eliminación de tarifas arancelarias beneficiaría a los países más pobres de la propia UE. En Estados Unidos, la eliminación de los aranceles sería beneficiosa sobre todo si se ampliara en el terreno de las barreras no arancelarias, con el resultado de un aumento de empleos del $13 \%$.

Mientras los cálculos generados no observan diferencias en la diversión del comercio a otros países, si se combinan la reducción de aranceles y los obstáculos no arancelarios, los ingresos en el mundo aumentarían en un $3.2 \%$. En suma, habría beneficios de aumento de empleos y descenso del desempleo en Estados Unidos, la Unión Europea y los demás países de la OCDE, sobre todo si el proyecto se ampliara a la reducción de los obstáculos no arancelarios. En términos simplistas, los puestos de trabajo en la UE

de enero de 2014. http://www.euractiv.com/eu-elections-2014/french-senators-violentlyattack-news-532705

10 Parlement Wallon, Session 2014-2015, Comité d'avis chargé des questions européennes sous la présidence de M. André Antoine, Président du Parlement, Jeudi 11 décembre 2014. Audition de M. Defraigne, Directeur de la Fondation Madariaga-Collège d'Europe et professeur au Collège d'Europe et à Sciences-Po Paris. Madariaga-College of Europe Foundation https://gallery.mailchimp.com/459b08cfdc09c91db4548094d/files/2014_12_11_ Pierre_Defraigne_Audition_Parlement_wallon.pdf

11 FELBERMAYR, G. et al. 2013. 'Transatlantic Trade and Investment Partnership (TTIP): Who benefits from a free trade deal? Part 1: Macroeconomic Effects», Bertelsmann Stiftung, 17 de junio de 2013, http://www.bfna.org/sites/default/files/TTIP-GED\%20 study\%2017June\%202013.pdf 
aumentarían en 2 millones anualmente si el acuerdo solamente se ampliara a la reducción de los obstáculos no arancelarios, mientras si se limitara a los aranceles se crearían solamente medio millón. En general, el TTIP lograría un equilibrio de empleo en los países con desempleo mayor que la media a niveles de la OCDE.

\section{Reticencias de las posiciones españolas}

En el entramado político español, existe el consenso de que los dos grandes partidos están a favor del desarrollo del acuerdo. ${ }^{12}$ Pero se teme por la actitud y el papel opositor generados en el seno de los nuevos sectores que están presentando una política de competencia en el Parlamento. La izquierda radical española (tanto la tradicional centrada en Izquierda Unida y Plural, como la novedosa formación Podemos) basa su oposición al TTIP en su denuncia de la opacidad del proceso y la acusación de que reforzaría la grieta social, el desempleo y la desigualdad. Se aduce que en los inicios de la negociación, reducida a los aspectos puramente técnicos, no han estado presentes ni el Senado de Estados Unidos ni el Consejo de la UE, y mucho menos el Parlamento de la UE. Todas las negociaciones hasta finales de 2014, han sido conducidas por la Comisión Europea y el Departamento de Comercio norteamericano. Los observadores críticos señalan que si hubiera plena publicidad, la oposición al proyecto crecería al conocerse diversas dimensiones desconocidas o soslayadas.

Como base del desacuerdo, se acusa al TTIP de no ser en realidad un acuerdo comercial, sino que se centraría predominantemente en reducir hasta el 80\% las barreras no arancelarias. Esta es el área sensible ocupada por estándares y las normas para la fabricación de los productos que son el objeto del comercio. Este recorte se traduciría en devaluación de las condiciones sociales y ambientales que son terreno sólido de las garantías de la UE. Se acusa que los verdaderos protagonistas de las negociaciones son los lobbies a ambos lados del Atlántico.

En cuanto a las cifras de beneficios que se barajan, se señala que no se notarán hasta por lo menos el año 2027, siempre que se eliminen por lo menos la mitad de los obstáculos no arancelarios. En cuanto a los puestos de trabajo que se predice serán generados, no se tiene garantía de que los cambios en el tejido laboral serán positivos en todo el mundo. Finalmente se aduce que los gobiernos, la sociedad civil en general y las empresas no ten-

12 Para consulta sobre la información oficial de la Representación de la Comisión Europea en España, véase la página digital: http://ec.europa.eu/spain/sobre-la-ue/ttip/espana_ es.htm 
drán poder para demandar a una potente multinacional, protegida por las garantías del acuerdo. ${ }^{13}$

Sutiles comentarios críticos utilizan metáforas innovadoras como comparar el secretismo con el que se han llevado las negociaciones con el llamado «efecto Drácula», en la versión cinematográfica de Coppola basada en la novela de Bram Stokerde, que sería un vampiro que no soporta ver la luz del sol. No es posible que en una negociación total se aísle a la banca y la industria financiera. Es una apuesta de fracaso similar al del Acuerdo Multilateral de Inversiones (AMI), enterrado por la ausencia de transparencia. Otro problema es común al sufrido por las negociaciones con Canadá y México, al aceptarse unos acuerdos de mínimos y a la baja. ${ }^{14}$

En los medios parlamentarios españoles afines al PSOE se asume la prioridad de que el proyecto está íntimamente ligado con la necesidad de salir de la crisis económica, por lo que los socialistas no quisieran verse señalados como opositores de algo que en principio debiera crear empleo y reducir el paro. Pero son conscientes de que tanto en Europa como en Estados Unidos esas metas pueden contaminarse de los movimientos populistas (Tea Party y afines en Estados Unidos y Podemos en España). Existe temor de amenazas contra el estado de bienestar. En el terreno geopolítico, sectores de liderazgo en el PSOE ven en el proyecto ventajas y desventajas, con unos posibles perdedores: los países en desarrollo y los BRICS. Se preguntan también cuáles pueden ser las implicaciones en el entramado de la OTAN (contratos militares y concursos públicos). Aunque el tema central no se considera que sean los cambios de las regulaciones, hay preocupación acerca de los niveles de indemnización y los procedimientos de arbitraje.

Los sectores mediáticos más críticos reflejan un sentimiento generalizado que prudentemente no se revela en las declaraciones gubernamentales. Como se alude más arriba, se toma nota del hecho que el Congreso de Estados Unidos ha exigido al presidente Obama que haga los necesarios esfuerzos para lograr la llamada Autoridad de Promoción Comercial (TPA), o sea la credencial para negociar los tratados. En ese aspecto hay un acuerdo básico con la UE, ya que en Bruselas los funcionarios y los líderes quieren estar seguros que luego de aprobarse cualquier esquema del TTIP, no será modificado por el Congreso. Esta incertidumbre sería generada por la ale-

13 Entre los comentarios cautelosos o alarmantes, véanse: GALLEGO-DÍAZ, S., «Europa alboroza a Wall Street», El País, 6 de julio de 2014, http://elpais.com/ elpais/2014/07/04/opinion/1404469344_631877.html; GONZÁLEZ, A., «El acuerdo EE UUUE se empantana», El País, 20 de julio de 2024; La Vanguardia. «Luz sobre el TLC con Estados Unidos», 27 de julio de 2014, http://www.lavanguardia.mobi/slowdevice/opinion/ editorial/20140727/54412508155/luz-sobre-el-tlc-con-estados-unidos.html

14 ESTEFANÍA, J., «"Efecto Drácula” transatlántico», El País, 16 de junio de 2014. 
gación de la existencia de una autoridad superior, aunque a ambas orillas del Atlántico se acepte que el máximo representante del pueblo tenga una palabra final en la ratificación de estos acuerdos. Pero no se acepta trabajar bajo la amenaza de suspensión o modificación sustancial. Juncker, por su parte, ha declarado repetidamente que existen una «líneas rojas» europeas en temas de salud, seguridad, protección social, privacidad de datos, y diversidad cultural. No se sacrificarán los temas que se consideran conquistas europeas fundamentales.

Con la preparación técnica ya muy avanzada, solamente faltaría la decisión política, pero todavía quedarían problemas por resolver. El primero sería la necesidad de llegar a un «reconocimiento mutuo» de fabricación, a una identificación de equivalencias en sectores sensibles como el automotor o los concursos en productos farmacéuticos, la ausencia de discriminación en contratos públicos, sobre todo en sectores de gran beneficio, como son los aeropuertos, puertos, y diversas infraestructuras, donde la competividad europea es notable. Europa espera que el lema de «Buy American» se traduzca en un «Buy Transatlántico».

Como reflejo oficial del gobierno español, se han estado expresando el Ministerio de Asuntos Exteriores y de Cooperación y el de Comercio de España, brindando el apoyo incondicional a la Comisión Europea. Por una parte, se entienden los reparos sobre la difusión pública de los detalles de la negociación. Se insta a ambos socios a hallar una solución para los desacuerdos iniciales. Pero hay una preocupación por la posible ralentización del proceso debida a una incapacidad de prevenir problemas. En esta primera fase, el gobierno español se ha mostrado satisfecho por concentrarse en solamente los temas de aranceles y servicios, y se registra una preocupación por las reticencias de Estados Unidos en el terreno energético.

\section{Percepción de Estados Unidos}

\section{El factor intrahistórico}

Los negociadores y observadores europeos debieran prestar atención a un perfil complejo de la identidad norteamericana que frecuentemente confunde cuando el análisis está afectado por el estereotipo y el perfil unidimensional. Más que la sucesión de los hechos que muestra la historia, convendría meditar sobre lo que Unamuno llamaba la «intrahistoria». Tal como se ha aludido más arriba, Estados Unidos, a pesar de lo que dé a entender la actuación del momento, se ha estado moviendo en los aledaños del intervencionismo, pero siempre ha estado anclado en su esencial aislacionismo, que regresa de forma contumaz en cualquier recodo del camino. 
En cualquier negociación con Europa, conviene recordar que Estados Unidos es un «país» (mejor: una idea) europeo. Pero su obsesión ha oscilado entre los extremos de ignorar a Europa, superarla y en ciertos momentos rescatarla. Pero el sentimiento más fuerte es resaltar el origen de rechazar ser Europa. Recuérdese la acusación de Romney contra Obama de tratar de convertir a Estados Unidos en «un país europeo» por sentirse inclinado en adoptar algunas conquistas del estado de bienestar, la emblemática seña de identidad europea.

El contexto de Estados Unidos está dominado por la actitud que pueda tomar no solamente el Partido Republicano, abierto en principio a la apertura comercial y de inversiones, sino también al impredecible papel que pueda jugar el Tea Party, o mejor dicho sus miembros independientes, cada uno atento a sus conveniencias locales. En general, habrá que contar con las tendencias proteccionistas de ciertos sectores tradicionalmente sostenedores del poder del Partido Demócrata, donde los sindicatos pueden mostrar actitudes cerradas, al mismo tiempo que se pueden ver atraídos por las ventajas laborales que los estándares europeos les pueden proporcionar.

En cualquier etapa de las discusiones ese sentimiento intrahistórico puede salir a la superficie. De la misma manera que se reacciona ante la supuesta «invasión» de los jóvenes latinoamericanos en el territorio norteamericano con un cierre de fronteras, la cesión de privilegios en el TTIP se puede aducir como rendición ante Europa.

Conviene situar estos argumentos en el contexto de la controversia sobre la política exterior de Obama en el marco del recrudecimiento de las tensiones como resultado de la crisis de Ucrania, el deterioro político-social de Libia, la inestabilidad de Egipto, la amenazante desintegración de Irak, la irresolución del conflicto en Siria, y finalmente el surgimiento del Estado Islámico (ISIS). El mensaje del presidente Obama, explícito desde su discurso en West Point, es reafirmar la «indispensabilidad» de Estados Unidos, pero no limitando su actuación exterior al terreno militar. En cierta manera, la nueva actitud responde al reposicionamiento a una visión muy alejada al intervencionismo y que respondería al sentimiento generalizado en la ciudadanía norteamericana hacia un novedoso y prudente aislacionismo. En este contexto, una confusa negociación el TTIP y las sospechas de reducción de sus ventajas pueden contribuir a una mayor ralentización del proceso, cuando no a su abandono.

\section{Entre la visión gubernamental y la analítica}

Las autoridades norteamericanas han admitido desde el principio que el acuerdo tiene una serie de prioridades. Destacan, por orden, algunas princi- 
pales: lograr el acceso al mercado abierto de la UE, aumentar considerablemente el volumen de las inversiones, eliminar totalmente los aranceles, lograr penetrar el sector de los servicios, reducir los obstáculos impuestos por las reglamentaciones, prestar atención a las pequeñas y medianas empresas, y situar el proyecto en el contexto de una agenda global, ampliable a otras regiones del planeta.

La comunidad de los think tanks (tan influyentes en Estados Unidos) llega al consenso de que, en realidad, el proyecto del TTIP no es una novedad en la evolución de las relaciones entre Estados Unidos y Europa ni en la agenda general del comercio mundial. ${ }^{15}$ El espíritu del acuerdo en ciernes estaba presente durante todo el periodo desde el final de la II Guerra Mundial, y muy especialmente desde el nacimiento y desarrollo de la propia UE. El debate ahora es si el esquema es demasiado limitado o, por el contrario, demasiado grande. La pregunta es si vale la pena plantearlo solamente o predominantemente sobre los obstáculos arancelarios. Esta duda se contesta con el argumento de que el volumen de las transferencias a ambos lados del Atlántico es ya tan enorme que solamente ese aspecto de eliminación ya lo convierten en atractivo y candidato para tener un impacto notable. Pero de todas maneras, la idea no es reducirlo al comercio, sino usarlo para reconstruir el partenariado (se llame alianza o asociación). ${ }^{16}$

Se cree que la dimensión esencialmente económica se derramará a otras zonas o áreas del globo. Más que un proyecto comercial, es un asunto estratégico, aspecto plenamente reconocido en vaga terminología, pero asumido con discreción. En la escena ampliada están presentes los poderes emergentes y el papel del multilateralismo, y numerosos observadores se preguntan si debiera estar abierto a otros actores ajenos a la estricta área nord-atlántica. Por ejemplo, las reacciones no se han hecho esperar en Turquía, y la preocupación ha surgido con respecto al impacto en los países en vías de desarrollo, y también los menos desarrollados en la propia Europa.

15 Véase el completo panorama ofrecido por la compilación del Center for Transatlantic Relations, The Paul H. Nitze School of Advanced International Studies, The Johns Hopkins University. TTIP and the Transatlantic Economy. 2014, http://transatlantic.sais-jhu.edu/, http://transatlantic.sais-jhu.edu/transatlantic-topics/transatlantic-economy-series.htm

16 HAMILTON, D., «The Geostrategic Aspects of a Transatlantic Trade and Investment Partnership», Center for Transatlantic Relations, Brookings Institution Press,John Hopkins University SAIS, 2014, http://transatlantic.sais-jhu.edu/publications/books/The\%20Geopolitics\%20of\%20TTIP/Hamilton_TTIP_geopolitics_summary_final.pdf; KUPCHAN, C., «The Geopolitical Implications of the Transatlantic Trade and Investment Partnership», German Marshall Fund, junio de 2014, http://www.gmfus.org/archives/the-geopolitical-implicationsof-the-transatlantic-trade-and-investment-partnership/ 
De todas maneras, no hay que engañarse: los problemas surgirán con contundencia. Por ejemplo, algunos conflictos serán provocados por las divergencias en el sector agrario. Habrá confrontaciones en el terreno financiero (si se incluye). Entre los temas espinosos geopolíticos habrá que sopesar el impacto de crisis como la de Ucrania, el desafío islamista en Irak y Siria, y la sempiterna agenda de la especificidad de seguridad nacional en Estados Unidos. En cualquier momento, un capítulo final de las negociaciones se puede empantanar por la intromisión de un aspecto político, manipulable en los medios de comunicación por motivaciones populistas. Finalmente, se habrá de tener en cuenta el calendario que ha comenzado con los resultados de las elecciones al Parlamento Europeo y la reconstitución de los puestos de decisión de la propia UE, las elecciones intermedias en Estados Unidos y la campaña para la captura de la Casa Blanca. De todas maneras, la agenda es ambiciosa y el capital político ya invertido es cuantioso. Es posible, siguiendo la metáfora plasmada por el Departamento de Comercio norteamericano, que no se tenga suficiente con usar «un depósito de gasolina», sino que se necesite repostar en otra gasolinera más de una vez.

La pregunta central, con un foco más economicista, es: ¿qué se puede esperar del TTIP? El objetivo parece estar claro: reforzar el crecimiento económico y el empleo. La base ya existe: el comercio de bienes y servicios supera el trillón de dólares anualmente, y los cuatro trillones de dólares en inversiones directas. El TTIP planea eliminar los aranceles y reducir sustancialmente las barreras no arancelarias en comercio e inversiones. Además, el acuerdo incluye otras metas ambiciosas como la coordinación y/o la armonización de las políticas regulatorias que afectan al comercio de bienes y servicios. La liberación de los servicios y manufacturas es también esencial para reforzar la competitividad y la participación en las cadenas de valor global. Las provisiones del TTIP en el sector de servicios, en las empresas estatales, y en los temas de medio ambiente, laborales y de políticas de competencia pueden sentar precedentes para establecer estándares globales y planear las futuras negociaciones de la OMC. Además, otra contribución novedosa a las reglas comerciales será la convergencia de las regulaciones. ${ }^{17}$

Entre los problemas y los retos de la aprobación y ejecución del acuerdo destaca la fuerte oposición política de las agencias regulatorias independientes, además de las diferencias de Estados Unidos y Europa al tratar las políticas regulatorias financieras. No será fácil reducir los aran-

17 SCHOTT, J., Broad Objectives and Prospects for TTIP, Peterson Institute for International Economics, 1 de mayo de 2014, http://www.piie.com/publications/papers/ schott20140501ppt.pdf 
celes agrarios sin también recortar los subsidios. Más difícil resultará el acceso a las contratas de los gobiernos estatales y locales en Estados Unidos y a las empresas públicas en Europa. En su momento, se evidenciarán las preocupaciones del público sobre el sistema de resolución de disputas entre inversores y gobiernos estatales y los temas sensibles de privacidad de datos. ${ }^{18}$

\section{Repercusiones en América Latina}

En cuanto al impacto que el acuerdo entre la UE y EE.UU. pueda tener en América Latina, debe razonablemente abandonarse la tentación de creer que los países latinoamericanos están inmunes a los efectos que el TTIP pueda tener en su entorno. La globalización, fuerza motriz del propio proyecto bicontinental, no permite excepciones. En consecuencia, no cabe la menor duda de que las repercusiones de este proyecto se notarán en América en un abanico de avenidas, cada una de ellas complementarias de las demás. Se recomienda, por lo tanto, prestar atención a, por lo menos, tres ejes de actividades. El primero es el impacto directo en unos países selectivos, sobre todo los que ya tienen o tendrían acuerdos sólidos de cooperación económica con la UE o Estados Unidos. El segundo sería el impacto en esos propios acuerdos bi-regionales. ¿Cuál será el efecto del TTIP? ¿Los modificará, los reforzará o simplemente los amenazará negativamente? El tercer terreno de actividades estaría comprendido por los efectos en los mismos sistemas de integración económica o política en el mismo entramado interno latinoamericano.

En cuando al primer eje, todo depende de las reglamentaciones que cada uno de los esquemas presentan en la actualidad y las que tengan en el futuro. Dos alternativas están abiertas: la construcción de unos parape-

18 Como muestras de los análisis que reflejan un contraste de actitudes con respecto a ciertas dimensiones del proyecto, véanse: ATLANTIC COUNCIL, «TTIP on Track but off Message?», marzo de 2014. http://www.atlanticcouncil.org/publications/reports/ttip-ontrack-but-off-message; BLEIBERG, J. and WEST, D., «Threats to the Future of Cloud Computing: Surveillance and Transatlantic Partnership», Brookings Institution - Center on the United States and Europe, 17 de julio de 2014, http://www.brookings.edu/blogs/techtank/ posts/2014/07/17-cloud-computing-regulation-ttip; BROMUND, T., et al., «Transatlantic Trade and Investment Partnership (TTIP): Pitfalls and Promises», The Heritage Foundation, Issue Brief \# 4100 on Trade, 5 de diciembre de 2013. http://www.heritage.org/research/ reports/2013/12/transatlantic-trade-and-investment-partnership-ttip-pitfalls-and-promises; BRUCE, S., «Faith and skepticism about trade, foreign investment», Pew Research Center, 16 de septiembre de 2014, http://www.pewglobal.org/files/2014/09/Pew-Research-CenterTrade-Report-FINAL-September-16-2014.pdf 
tos que aíslen los acuerdos entre regiones, o dejarlo a la inercia del propio desarrollo de las fuerzas económicas. Con referencia al segundo, habrá que esperar a ver cuál es el desarrollo de las dos variantes y cuál tiene más poder de derrame.

Mucho más interesante e intrigante es el tercer escenario. Simplificando la consideración, los efectos del TTIP (igual que los más modestos del TIP en el contexto del Pacífico) pueden afectar a los sistemas de integración o cooperación económica latinoamericanos en dos casos extremos. Uno es el del «efecto refuerzo», como incentivo a la profundización de los diversos experimentos de integración, sobre todo los que son más exitosos o tienen más posibilidades de serlo. El otro es el contrario efecto de disgregación que favorecería la ejecución de las alternativas que ya ha sido señalada tanto por Estados Unidos como la UE: los acuerdos individuales y la consolidación del modelo NAFTA reducido al libre comercio. Esta alternativa representaría el triunfo definitivo del modelo norteamericano. Depende, por lo tanto, de la decisión política del liderazgo latinoamericano para optar por la disgregación o la unión para presentar alternativas y cooperación al imponente eje presentado por Bruselas y Washington. Solamente el paso de un tiempo prudencial dará el veredicto. ${ }^{19}$

Profundizando sobre la primera alternativa, que no depende exclusivamente de la decisión de los propios bloques latinoamericanos, requeriría una buena dosis de voluntad política en cada uno de los contextos, una cualidad que hasta el momento se ha presentado como muy débil. Considerados por separado, las predicciones oscilan entre un cierto optimismo en el caso de los entramados menores (Centroamérica, CARICOM) hasta los más aquejados de dudas con los de mayor extensión (MERCOSUR), con una predicción de progresiva desaparición con respecto a otros de cuya existencia en el futuro se tienen serias dudas (Comunidad Andina). Ya el Sistema Centroamericano de Integración (SICA) se convirtió en el aparente ganador de los favores de la Unión Europea con la firma del Acuerdo de Asociación, ansiado durante décadas.

A pesar de no contar con una sólida organización estructural de carácter unitario y supranacional, el SICA y los países miembros han conseguido

19 Véanse: PEÑA, F., «A World of Mega-interregional preferential spaces? Incentives for a more effective cooperation between Latin American countries», International Trade Relations Newsletter, Mayo de 2014, http://www.felixpena.com.ar/index.php?contenido=negot iations\&neagno=report/2014-05-incentives-more-effective-cooperation-between-latin-american-countries; PENAA, F., «The Negotiation of the TTIP: a Preliminary View from the Deep South» en ROY and DOMÍNGUEZ (eds), The TTIP:The Transatlantic Trade and Investment Partnership between the European Union and the United States, Miami-Florida European Union Center/Jean Monnet Chair, 2014, pp. 185-196. http://www.as.miami.edu/media/ college-of-arts-and-sciences/content-assets/euc/docs/books/TTIP.pdf 
una notable atención de la UE, que les pueden ayudar a reforzar su propia cohesión. Debido a sus dimensiones menores, el acuerdo entre la UE y Estados Unidos debe ayudar, más que perjudicar. Diferente el caso de CARICOM, cuyos destinos están afectados en mayor grado por la esquizofrenia de su lealtad hacia Europa (y sus antiguas metrópolis) y la irresistible atracción y presión de Estados Unidos. El TTIP impactará en CARICOM, pero es difícil predecir su impacto en la propia integración regional. Primero se deberá solidificar y clarificar la propia relación con la UE, el papel de CARIFORUM, y las internas disputas entre los países miembros de la Commonwealth y los demás vecinos.

El desafío que el TTIP presenta a MERCOSUR es formidable. Por un lado, destaca la actividad individual de Brasil, potencia que en todo momento calcula sus movimientos según sus intereses nacionales, frecuentemente no coincidentes con los bloques a los que pertenece. El hecho de ser un líder de los BRICS complica más el panorama. El detalle de estar tomando la iniciativa para conseguir un acuerdo autónomo con la UE, ya de por sí debilita a MERCOSUR, donde rivaliza en maniobras independientes con Argentina. El ingreso de Venezuela, cada vez más afectada por su crisis interna (no solamente política, sino también económica) ha convertido en dudoso el propio proceso de profundización de la integración sureña, a la vez de insertar una incógnita notable en las eternas negociaciones con la UE para la firma de su propio acuerdo, cuyo origen se remonta a más de dos décadas. De ser modelo de reproducción de la UE en el continente americano, MERCOSUR es hoy una molestia para Bruselas. Pero con la débil perspectiva de ofrecer un atractivo al futuro bloque entre la UE y Estados Unidos, la sensación de orfandad puede impulsar a los líderes mercosureños a superar sus propias carencias y reforzar su propia unidad. ${ }^{20}$

Finalmente, conviene hacer una escala en cuanto al impacto en la Comunidad Andina. A pesar de la necesidad acuciante de su refuerzo en una era de crecimiento del desarrollo de mega-acuerdos, el potencial de apuntalamiento de la CAN es dudoso. El organismo (cuya Secretaría General está huérfana de autonomía supranacional) no consigue ponerse de acuerdo para entablar negociaciones conjuntas con la UE. Su tejido de integración está zapado de la amenaza de Bolivia de abandonarlo, siguiendo la senda de Venezuela para insertarse en MERCOSUR. Perú y Colombia han firmado

20 MALAMUD, C., «The TTIP and Mercosur», en ROY and DOMÍNGUEZ (eds), The TTIP: The Transatlantic Trade and Investment Partnership between the European Union and the United States, Miami-Florida European Union Center/Jean Monnet Chair, 2014, pp. 173184. http://www .as.miami.edu/media/college-of-arts-and-sciences/content-assets/euc/docs/ books/TTIP.pdf 
acuerdos beneficiosos tanto con la UE como con Estados Unidos (lo cual es ya una muestra del cuestionamiento de la validez de la CAN). Ecuador simplemente se mantiene aislado, aunque fiel a los principios bolivarianos, en vías de deterioro. Más posibilidades se le presentan en la evolución del TIP del escenario del Pacífico.

Naturalmente, la propia ALBA (ya de por sí debilitada por los problemas de Venezuela) no existe como tal en el terreno de acuerdos como el TTIP. En ese contexto ideológico, conviene anotar que desde perspectivas de ultraizquierda se han acrecentado los análisis que identifican el proyecto del TTIP como una nueva versión de «neoimperialismo» de origen norteamericano, ahora en sintonía con Estados Unidos. Esta identificación ejercerá una presión en los gobiernos latinoamericanos (no solamente miembros de pleno derecho de ALBA) que opten por esa identificación, lo cual los alejarán de posibles tratos beneficiosos

Finalmente, hay que admitir que el desarrollo del TTIP no tendrá efecto alguno, ni se verá afectado en negativo, a través de la acción de los improbables frentes comunes de perfil inter-latinoamericano como UNASUR. Solamente la CELAC tiene una mínima consistencia en presentarse como interlocutora, mediante los acuerdos entablados en sus cumbres bianuales, de los débiles intereses conjuntos del conglomerado latinoamericano y caribeño. Para dialogar con serias expectativas de éxito con Estados Unidos y la UE se debe contar con un teléfono al modo de Kissinger, aunque se crea que Bruselas no lo tiene.

\section{Brevísima y provisional conclusión}

Tal como se ha delineado en el cuerpo de este informe, la evolución y eventual ejecución del acuerdo, sea cual sea su forma, dependerá de un conjunto de detalles y circunstancias extremadamente complejas que se desarrollarán a lo largo de 2015 y probablemente quizá más allá. En síntesis, dependerá de un conjunto de percepciones que deberán compactarse y complementarse para producir un mínimo de éxito. En primer lugar, las ambivalentes actitudes de los sectores de Estados Unidos que se sienten reacios pueden asentir en que el acuerdo es en el fondo una extensión de los beneficios del mercado único europeo al otro lado del Atlántico. En el bando europeo, una vez se decida dejar de lado ciertos aspectos que no son negociables, la opinión pública y los responsables políticos deberán aceptar un mínimo de mutuo reconocimiento de regulaciones, tal como se consiguió a lo largo del proceso europeo en aceptar las normas nacionales de los países que se adherían a la integración. La imagen del acuerdo como un caballo de Troya debe desterrarse. Bruselas y Washington deberán suavizar sus re- 
clamos y no insistir tanto en los especulativos beneficios de crecimiento y producción de empleos. Se aconseja que los mensajes hacia sus respectivos clientes electorales y poderes económicos debieran enfatizar las ventajas inherentes de la alianza en ciernes. Se trata de dos socios naturales que ya comparten características innatas en sus valores, aspectos por demostrar en otro tipo de componendas y tratos con los poderes (emergentes o no) en otros continentes. ${ }^{21}$ La pregunta fundamental debiera ser: ¿cuál es la alternativa a este acuerdo?

${ }^{21}$ Como resumen de las advertencias presentes en ambos bandos, véase este análisis: THE ECONOMIST, Charlemagne, «Ships that pass in the night», 13 de diciembre de 2014, p. 57. http://www.economist.com/news/europe/21636061-trade-deal-america-would-be-goodeverybody-yet-it-still-may-not-happen-ships-pass 
